\title{
Periodic boundary value problems for first-order impulsive difference equations with time delay
}

\author{
Jingfeng Tian', Wenli Wang ${ }^{2}$ and Wing-Sum Cheung ${ }^{3 *}$ (i)
}

\author{
"Correspondence: \\ wscheung@hku.hk \\ ${ }^{3}$ Department of Mathematics, \\ University of Hong Kong, Pokfulam, \\ Hong Kong \\ Full list of author information is \\ available at the end of the article
}

\begin{abstract}
This paper focuses on a certain type of periodic boundary value problems for first-order impulsive difference equations with time delay. Notions of lower and upper solutions are introduced, with which two new comparison theorems are established. Using Schaefer's fixed point theorem, sufficient conditions for the existence and uniqueness of solutions to the corresponding linear problem of the boundary value problem are derived. By utilizing monotone iterative methods combined with the methods of lower and upper solutions, an existence theorem of extremal solutions to first-order impulsive difference equations with delay is obtained. These results extend some existing results in the literature. An interesting example is also given to verify the results obtained.
\end{abstract}

MSC: 39A10; 34B37

Keywords: Impulsive difference equations; Time delay; Comparison principle; Periodic boundary value problem; Extremal solutions

\section{Introduction}

The mathematical model of many real-world phenomena can be represented by impulsive equations, and these phenomena have undergone significant changes in the progress. Such equations have a wide range of applications in many areas, including economics, optimal control, dynamic systems, medicine, and many other fields (see [1-6]). In the past decade, there has been an increasing interest in extending impulsive differential equations to timedelay systems and boundary value problems. For example, in 2003, De la Sen and Luo [7] studied the stability of a class of linear time-delay systems. Immediately after that, De la Sen discussed in [8] the time-varying systems with non-necessarily bounded everywhere continuous time-differentiable time-varying point delays. Wang and Ding [9] considered asymptotic stability and exponential stability of impulsive control systems with delay. Zhao et al. [10] extended the method of lower and upper solutions to the framework of $m$-point impulsive boundary value problem. Interested readers may consult the monograph [11] for more details on impulsive differential equations.

Research in difference equations has been active in recent years and has played an important role in numeral fields, including biology, computing, electrical circuit analysis, etc. (see [12-14]). There also has been a large number of studies on the extension of difference

(c) The Author(s) 2018. This article is distributed under the terms of the Creative Commons Attribution 4.0 International License (http://creativecommons.org/licenses/by/4.0/), which permits unrestricted use, distribution, and reproduction in any medium, provided you give appropriate credit to the original author(s) and the source, provide a link to the Creative Commons license, and indicate if changes were made. 
equations to time-delay systems in the literature [15-24]. Among these extensions, Zhu et al. [23] studied impulsive delay difference equations and gave the results of global exponential stability, and Li and Song [17] studied the asymptotic behavior of impulsive delay difference equations. However, there are not many related results for impulsive difference equations and impulsive delay difference equations. Based on the above observation, in this paper, periodic boundary value problems for impulsive difference equations with time delay are considered.

To state our results, we first introduce the following symbols:

$J:=Z[0, N]=\{0,1, \ldots, N\}$, where $N \in \mathbb{N}$ is a positive integer;

$J^{\prime}:=Z[0, N-1]=\{0,1, \ldots, N-1\}$

$\Omega:=$ the collection of $\mathbb{R}$-valued functions defined on $J$; and

$\|y\|:=\max _{k \in J}|y(k)|$ for any $y \in \Omega$.

We will consider the following first-order impulsive difference equations with time delay:

$$
\left\{\begin{array}{l}
\Delta y(k)=g(k, y(k), y(\theta(k))), \quad k \neq k_{\tau}, k \in J^{\prime}, \\
\Delta y\left(k_{\tau}\right)=I_{\tau}\left(y\left(k_{\tau}\right)\right), \quad \tau=1,2, \ldots, m, \\
y(0)=y(N),
\end{array}\right.
$$

where $\Delta y(k)=y(k+1)-y(k), g \in C\left(J \times \mathbb{R}^{2}, \mathbb{R}\right), \theta \in C(J, Z[0, k]), 0 \leq \theta(k) \leq k, I_{\tau} \in C(\mathbb{R}, \mathbb{R})$ $(\tau=1,2, \ldots, m)$, and $0<k_{1}<k_{2}<\cdots<k_{m}<N$.

We note that when $\theta(k)=k$, problem (1.1) is reduced to periodic boundary conditions for impulsive equations, which was studied in [16].

When $\theta(k)=k$ and $I_{\tau}(x) \equiv 0, \tau=1,2, \ldots, m$, problem (1.1) does not contain the impulse term, and they become ordinary difference equations, which have been investigated extensively by many authors $[6,24]$.

This paper is organized as follows. In Sect. 2, new definitions of lower and upper solutions are given, and two new comparison theorems are proved. In Sect. 3, the existence and uniqueness of solutions to the linear problem associated with (1.1) are established. Then, utilizing the monotone iterative method combined with the method of lower and upper solutions, problem (1.1) is proved to have extremal solutions. Finally, an example is given to document the results obtained.

\section{Comparison results}

To apply the monotone iterative method to problem (1.1), we first introduce some definitions and lemmas.

Definition 2.1 A function $v \in \Omega$ is said to be a lower solution of (1.1) if there exist $H \geq 0$ and $0<L \leq M_{\tau}<1$ such that

$$
\left\{\begin{array}{l}
\Delta v(k) \leq g(k, v(k), v(\theta(k)))-r_{v}, \quad k \neq k_{\tau}, k \in J^{\prime}, \\
\Delta v\left(k_{\tau}\right) \leq I_{\tau}\left(v\left(k_{\tau}\right)\right)-d_{\nu \tau}, \quad \tau=1,2, \ldots, m,
\end{array}\right.
$$

where

$$
r_{v}= \begin{cases}\frac{L k+H \theta(k)+1}{N}[v(0)-v(N)], & \text { if } v(0)>v(N), \\ 0, & \text { if } v(0) \leq v(N),\end{cases}
$$




$$
d_{v \tau}= \begin{cases}\frac{M_{\tau} k_{\tau}+1}{T}[v(0)-v(N)], & \text { if } v(0)>v(N) \\ 0, & \text { if } v(0) \leq v(N)\end{cases}
$$

Definition 2.2 A function $w \in \Omega$ is said to be an upper solution of (1.1) if there exist $H \geq 0$ and $0<L \leq M_{\tau}<1$ such that

$$
\left\{\begin{array}{l}
\Delta w(k) \geq g(k, w(k), w(\theta(k)))+r_{w}, \quad k \neq k_{\tau}, k \in J^{\prime} \\
\Delta w\left(k_{\tau}\right) \geq I_{\tau}\left(w\left(k_{\tau}\right)\right)+d_{w \tau}, \quad \tau=1,2, \ldots, m
\end{array}\right.
$$

where

$$
\begin{aligned}
& r_{w}= \begin{cases}\frac{L k+H \theta(k)+1}{N}[w(N)-w(0)], & \text { if } w(0)<w(N), \\
0, & \text { if } w(0) \geq w(N),\end{cases} \\
& d_{w \tau}= \begin{cases}\frac{M_{\tau} k_{\tau}+1}{N}[w(N)-w(0)], & \text { if } w(0)<w(N), \\
0, & \text { if } w(0) \geq w(N) .\end{cases}
\end{aligned}
$$

Lemma 2.3 (Discrete Theorem 1.4.1 [16]) Let $\left\{l_{k}\right\}$ and $\left\{q_{k}\right\}$ be two real sequences with $l_{k}>-1$ for all $k$, and $a_{\tau}, b_{\tau}$ be constants with $a_{\tau} \geq 0$ for all $\tau$. Assume that

(i) $\left\{k_{\tau}\right\}$ is a sequence satisfying $0 \leq k_{0}<k_{1}<\cdots<k_{\tau}<\cdots$, and $\lim _{\tau \rightarrow \infty} k_{\tau}=\infty$;

(ii) for $\tau \in \mathbb{N}, k \geq k_{0}$,

$$
\left\{\begin{array}{l}
\Delta p(k) \leq l_{k} p(k)+q_{k}, \quad k \neq k_{\tau} \\
p\left(k_{\tau}+1\right) \leq a_{\tau} p\left(k_{\tau}\right)+b_{\tau}
\end{array}\right.
$$

Then

$$
\begin{aligned}
p(k) \leq & p\left(k_{0}\right) \prod_{k_{0}<k_{\tau}<k} a_{\tau} \prod_{k_{0}<j<k, j \neq k_{\tau}, \tau \in \mathbb{N}}\left(1+l_{j}\right) \\
& +\sum_{j=k_{0}, j \neq k_{\tau}}^{k-1} \prod_{j<k_{\tau}<k} a_{\tau} \prod_{j<s<k, s \neq k_{\tau}}\left(1+l_{s}\right) q_{j} \\
& +\sum_{k_{0}<k_{\tau}<k} b_{\tau} \prod_{k_{\tau}<k_{j}<k} a_{j} \prod_{k_{\tau}<j<k, j \neq k_{j}, j \in \mathbb{N}}\left(1+l_{j}\right) .
\end{aligned}
$$

Lemma 2.4 (Theorem $2.2[20]$ ) Assume that $p \in \Omega$ satisfies

$$
\left\{\begin{array}{l}
\Delta p(k)+L p(k)+H p(\theta(k)) \leq 0, \quad k \neq k_{\tau}, k \in J^{\prime} \\
\Delta p\left(k_{\tau}\right) \leq-M_{\tau} p\left(k_{\tau}\right), \quad \tau=1,2, \ldots, m \\
p(0) \leq 0
\end{array}\right.
$$

where $H \geq 0,0<L \leq M_{\tau}<1$ for $\tau=1,2, \ldots, m$, and

$$
H \sum_{i=0, i \neq k_{\tau}}^{N} \prod_{i<k_{\tau}<k}\left(1-M_{\tau}\right)(1-L)^{\theta(i)-i-1}-\prod_{\tau=1}^{m}\left(1-M_{\tau}\right) \leq 0 .
$$

Then $p(k) \leq 0, k \in J$. 
Lemma 2.5 Assume that (2.1) holds and $p \in \Omega$ satisfies

$$
\left\{\begin{array}{l}
\Delta p(k)+L p(k)+H p(\theta(k)) \leq-r_{p}, \quad k \neq k_{\tau}, k \in J^{\prime} \\
\Delta p\left(k_{\tau}\right) \leq-M_{\tau} p\left(k_{\tau}\right)-d_{p \tau}, \quad \tau=1,2, \ldots, m,
\end{array}\right.
$$

where $H \geq 0,0<L \leq M_{\tau}<1$ for $\tau=1,2, \ldots, m$, and

$$
\begin{aligned}
& r_{p}= \begin{cases}\frac{L k+H \theta(k)+1}{N}[p(0)-p(N)], & \text { if } p(0)>p(N), \\
0, & \text { if } p(0) \leq p(N),\end{cases} \\
& d_{p \tau}= \begin{cases}\frac{M_{\tau} k_{\tau}+1}{N}[p(0)-p(N)], & \text { if } p(0)>p(N), \\
0, & \text { if } p(0) \leq p(N) .\end{cases}
\end{aligned}
$$

Then $p(k) \leq 0$ on $J$.

Proof Suppose that the conclusion is not true, then $p(k)>0$ for some $k \in J$. There are two cases as follows.

Case 1: $p(0) \leq p(N)$. Let $q(k)=(1-M)^{-k} p(k)$ for $k \in J$. Then $q(k)$ satisfies

$$
\left\{\begin{array}{l}
\Delta q(k) \leq-H(1-L)^{\theta(k)-k-1} q(\theta(k)), \quad k \neq k_{\tau}, k \in J^{\prime} \\
\Delta q\left(k_{\tau}\right) \leq \frac{L-M_{\tau}}{1-L} q\left(k_{\tau}\right), \quad \tau=1,2, \ldots, m, \\
q(0) \leq(1-L)^{N} q(N) .
\end{array}\right.
$$

It is easily seen that we only need to prove $q(k) \leq 0$ for $k \in J$. Assume that it is not true. Then there are two sub-cases:

(1) There exists $k^{*} \in J$ such that $q\left(k^{*}\right)>0$, and $q(k) \geq 0$ for $k \in J$;

(2) There exists $k^{*} \in J$ such that $q\left(k^{*}\right)<0$.

In sub-case (1), (2.2) implies that

$$
\left\{\begin{array}{l}
\Delta q(k) \leq 0, \quad k \neq k_{\tau}, k \in J^{\prime} \\
\Delta q\left(k_{\tau}\right) \leq 0, \quad \tau=1,2, \ldots, m
\end{array}\right.
$$

This means that $q(k)$ is a non-increasing function. Then we obtain $q(0) \geq q\left(k^{*}\right)$ and $q(0) \geq$ $q(N)>q(N)(1-L)^{N}$, which contradicts $(2.2)$.

In sub-case (2), set $\min _{k \in Z[0, N]} q(k)=-\lambda, \lambda \leq 0$. Without loss of generally, we can assume that there exists $k_{\tau}<\bar{k} \leq k_{\tau+1}$ for some $\tau$ such that $q(\bar{k})=-\lambda$ or $q\left(k_{\tau}\right)=-\lambda$. Assume that $q(\bar{k})=-\lambda$ (if $q\left(k_{\tau}\right)=-\lambda$, the proof is similar). From (2.2), we have

$$
\begin{aligned}
q(k) & \leq q(\bar{k}) \prod_{\bar{k}<k_{\tau}<k}(1-L)^{-1}\left(1-M_{\tau}\right)+\lambda H \sum_{j=\bar{k}, j \neq k_{\tau}}^{k-1} \prod_{i<k_{\tau}<k}\left(1-M_{\tau}\right)(1-L)^{\theta(j)-j-2} \\
& \leq-\lambda \prod_{\tau=1}^{m}(1-L)^{-1}\left(1-M_{\tau}\right)+\lambda H \sum_{j=0, j \neq k_{\tau}}^{N} \prod_{j<k_{\tau}<k}\left(1-M_{\tau}\right)(1-L)^{\theta(j)-j-2} .
\end{aligned}
$$


Putting $k=N$ in (2.3), we have

$$
\begin{aligned}
q(N) & \leq \lambda\left\{H \sum_{j=0, j \neq k_{\tau}}^{N} \prod_{j<k_{\tau}<k}\left(1-M_{\tau}\right)(1-L)^{\theta(j)-j-2}-\prod_{\tau=1}^{m}(1-L)^{-1}\left(1-M_{\tau}\right)\right\} \\
& \leq \lambda(1-L)^{-1}\left\{H \sum_{j=0, j \neq k_{\tau}}^{N} \prod_{j<k_{\tau}<k}\left(1-M_{\tau}\right)(1-L)^{\theta(j)-j-1}-\prod_{\tau=1}^{m}\left(1-M_{\tau}\right)\right\} \\
& \leq 0,
\end{aligned}
$$

which is a contradiction with $q(N)>0$, and so $p(0) \leq 0$. From Lemma 2.4, we have $p(k) \leq 0$, $k \in J$. Then the result follows.

Case 2: $p(0)>p(N)$. Let

$$
\bar{p}(k)=p(k)+h(k), \quad k \in J,
$$

where $h(k)=\frac{k}{N}[p(0)-p(N)], k \in J$. Then $h(0)=0, h(N)=p(0)-p(N)$, and $h(k) \geq 0$ on $J$. Hence we have

$$
\begin{aligned}
& \bar{p}(0)=p(0)=h(N)+h(N)= \bar{p}(N), \\
& \Delta \bar{p}(k)+L \bar{p}(k)+H \bar{p}(\theta(k))= \Delta p(k)+L p(k)+H p(\theta(k)) \\
&+\frac{L k+H \theta(k)+1}{N}[p(0)-p(N)] \\
& \leq 0, \quad k \neq k_{\tau}, \tau \in J^{\prime}, \\
& \Delta \bar{p}\left(k_{\tau}\right)=\Delta p\left(k_{\tau}\right)+\frac{1}{N}[p(0)-p(N)] \\
& \leq-M_{\tau} p\left(k_{\tau}\right)-\frac{M_{\tau} p_{\tau}+1}{N}[p(0)-p(N)]+\frac{1}{N}[p(0)-p(N)] \\
&=-M_{\tau} \bar{p}\left(k_{\tau}\right), \quad \tau=1,2, \ldots, m .
\end{aligned}
$$

By Case 1 , we have $\bar{p}(k) \leq 0, k \in J$. Then $p(k) \leq 0$ on $J$. The proof is finished.

\section{Existence results}

We consider the following linear problem associated with (1.1):

$$
\left\{\begin{array}{l}
\Delta y(k)+L y(k)+H y(\theta(k))=\sigma(k), \quad k \neq k_{\tau}, k \in J^{\prime}, \\
\Delta y\left(k_{\tau}\right)=-M_{\tau} y\left(k_{\tau}\right)+\gamma_{\tau}, \quad \tau=1,2, \ldots, m, \\
y(0)=y(N),
\end{array}\right.
$$

where $0<L \leq M_{\tau}<1, H \geq 0, \gamma_{\tau} \in \mathbb{R}(\tau=1,2, \ldots, m), \sigma \in C(J, \mathbb{R})$.

Definition 3.1 A function $v \in \Omega$ is said to be a lower solution of (3.1) if there exist $H \geq 0$ and $0<L \leq M_{\tau}<1$ such that

$$
\left\{\begin{array}{l}
\Delta v(k)+L v(k)+H v(\theta(k)) \leq \sigma(k)-r_{v}, \quad k \neq k_{\tau}, k \in J^{\prime} \\
\Delta v\left(k_{\tau}\right) \leq-M_{\tau} v\left(k_{\tau}\right)+\gamma_{\tau}-d_{\nu \tau}, \quad \tau=1,2, \ldots, m,
\end{array}\right.
$$


where

$$
\begin{aligned}
& r_{v}= \begin{cases}\frac{L k+H \theta(k)+1}{N}[v(0)-v(N)], & \text { if } v(0)>v(N), \\
0, & \text { if } v(0) \leq v(N),\end{cases} \\
& d_{v \tau}= \begin{cases}\frac{M_{\tau} k_{\tau}+1}{N}[v(0)-v(N)], & \text { if } v(0)>v(N), \\
0, & \text { if } v(0) \leq v(N),\end{cases}
\end{aligned}
$$

Definition 3.2 A function $w \in \Omega$ is said to be an upper solution of (3.1) if there exist $H \geq 0$ and $0<L \leq M_{\tau}<1$ such that

$$
\left\{\begin{array}{l}
\Delta w(k)+L w(k)+H w(\theta(k)) \geq \sigma(k)+r_{w}, \quad k \neq k_{\tau}, k \in J^{\prime} \\
\Delta w\left(k_{\tau}\right) \geq-M_{\tau} w\left(k_{\tau}\right)+\gamma_{\tau}+d_{w \tau}, \quad \tau=1,2, \ldots, m,
\end{array}\right.
$$

where

$$
\begin{aligned}
& r_{w}= \begin{cases}\frac{L k+H \theta(k)+1}{N}[w(N)-w(0)], & \text { if } w(0)<w(N), \\
0, & \text { if } w(0) \geq w(N),\end{cases} \\
& d_{w \tau}= \begin{cases}\frac{M_{\tau} k_{\tau}+1}{N}[w(N)-w(0)], & \text { if } w(0)<w(N), \\
0, & \text { if } w(0) \geq w(N) .\end{cases}
\end{aligned}
$$

Lemma 3.3 Let $0<L<1$. If a function $y \in \Omega$ is a solution of (3.1), then $y$ is a solution of the following equation:

$$
\begin{aligned}
y(k)= & \sum_{i=0, i \neq k_{\tau}}^{N-1} F(k, i)(\sigma(i)-H y(\theta(i))) \\
& +\sum_{0<k_{\tau} \leq N-1} F\left(k, k_{\tau}\right)\left[\left(L-M_{\tau}\right) y\left(k_{\tau}\right)+\gamma_{\tau}\right],
\end{aligned}
$$

where

$$
F(k, i)=\frac{1}{1-(1-L)^{N}} \begin{cases}\frac{(1-L)^{k}}{(1-L) i+1}, & 0 \leq i \leq k-1, \\ \frac{(1-L)^{N+k}}{(1-L)^{i+1}}, & k \leq i \leq N-1 .\end{cases}
$$

Proof Set $\bar{y}(k)=\frac{y(k)}{(1-L)^{k}}, k \in J$. From (3.1), we see that $\bar{y}(k)$ satisfies

$$
\left\{\begin{array}{l}
\bar{y}(k+1)=\bar{y}(k)+\frac{\sigma(k)-H \bar{y}(\theta(k))(1-L)^{\theta(k)}}{(1-L)^{k+1}}, \quad k \neq k_{\tau}, k \in J^{\prime}, \\
\Delta \bar{y}\left(k_{\tau}\right)=\frac{L-M_{\tau}}{1-L} \bar{y}\left(k_{\tau}\right)+\frac{\gamma_{\tau}}{(1-L)^{k_{\tau}+1}}, \quad \tau=1,2, \ldots, m, \\
\bar{y}(0)=\bar{y}(N)(1-L)^{N} .
\end{array}\right.
$$

By (3.3), one obtains

$$
\begin{aligned}
\bar{y}(k)= & \bar{y}(0)+\sum_{i=0, i \neq k_{\tau}}^{k-1} \frac{\sigma(i)-H \bar{y}(\theta(i))(1-L)^{\theta(i)}}{(1-L)^{i+1}} \\
& +\sum_{0<k_{\tau} \leq k-1}\left(\frac{L-M_{\tau}}{1-L} \bar{y}\left(k_{\tau}\right)+\frac{\gamma_{\tau}}{(1-L)^{k_{\tau}+1}}\right) .
\end{aligned}
$$


Letting $k=N$ in (3.4), we have

$$
\begin{aligned}
\bar{y}(N)= & \bar{y}(0)+\sum_{i=0, i \neq k_{\tau}}^{N-1} \frac{\sigma(i)-H \bar{y}(\theta(i))(1-L)^{\theta(i)}}{(1-L)^{i+1}} \\
& +\sum_{0<k_{\tau} \leq N-1}\left(\frac{L-M_{\tau}}{1-L} \bar{y}\left(k_{\tau}\right)+\frac{\gamma_{\tau}}{(1-L)^{k_{\tau}+1}}\right) .
\end{aligned}
$$

For $\bar{y}(N)=\frac{\bar{y}(0)}{(1-L)^{N}}$, and carrying out detailed computation, we obtain

$$
\begin{aligned}
\bar{y}(0)= & \frac{(1-L)^{N}}{1-(1-L)^{N}}\left[\sum_{i=0, i \neq k_{\tau}}^{N-1} \frac{\sigma(i)}{(1-L)^{i+1}}-H \bar{y}(\theta(i))(1-L)^{\theta(i)-i-1}\right. \\
& \left.+\sum_{0<k_{\tau} \leq N-1}\left(\frac{L-M_{\tau}}{1-L} \bar{y}\left(k_{\tau}\right)+\frac{\gamma_{\tau}}{(1-L)^{k_{\tau}+1}}\right)\right] .
\end{aligned}
$$

Combining (3.6) and (3.4), and applying $\bar{y}(k)=\frac{y(k)}{(1-L)^{k}}, k \in J$, we find that $y$ satisfies (3.2), hence $y$ is also a solution of (3.1).

Theorem 3.4 Let (2.1) hold with $H \geq 0,0<L \leq M_{\tau}<1(\tau=1,2, \ldots, m)$, and let $v, w \in \Omega$ be lower and upper solutions, respectively, of (3.1) with $v \leq w$ on J. Then problem (3.1) has a unique solution $y \in[v, w]=\{y \in \Omega: v(k) \leq y(k) \leq w(k), k \in J\}$.

Proof First, we consider the following equations:

$$
\left\{\begin{array}{l}
\Delta y(k)+L y(k)+H q(\theta(k), y(\theta(k)))=\sigma(k), \quad k \neq k_{\tau}, k \in J^{\prime}, \\
\Delta y\left(k_{\tau}\right)=-M_{\tau} q\left(k_{\tau}, y\left(k_{\tau}\right)\right)+\gamma_{\tau}, \quad \tau=1,2, \ldots, m, \\
y(0)=y(N),
\end{array}\right.
$$

where $q(k, r)=\max \{v(k), \min \{r, w(k)\}\}$ for $k \in J$, and $r \in \mathbb{R}$.

We define an operator $\phi$ by

$$
\begin{aligned}
{[\phi y](k)=} & \sum_{i=0, i \neq k_{\tau}}^{N-1} F(k, i)[\sigma(i)-H q(\theta(i), y(\theta(i)))] \\
& +\sum_{0<k_{\tau} \leq N-1} F\left(k, k_{\tau}\right)\left[\left(L-M_{\tau}\right) q\left(k_{\tau}, y\left(k_{\tau}\right)\right)+\gamma_{\tau}\right] .
\end{aligned}
$$

As $\sigma$ is continuous, $\phi: \Omega \rightarrow \Omega$ is continuous.

Also because $\sigma$ is continuous, we can select $l>0$ such that $|\sigma(k)| \leq h$, and let $h>0$ be such that $|v(k)| \leq h,|w(k)| \leq h, k \in J$. For $\lambda \in(0,1)$, any solution of

$$
y=\lambda \phi y
$$


satisfies

$$
\begin{aligned}
\|y\|= & \lambda\|\phi y\| \leq\|\phi y\| \\
\leq & \max _{k \in J} \sum_{i=0, i \neq k_{\tau}}^{N-1} F(k, i)|\sigma(i)-H q(\theta(i), y(\theta(i)))| \\
& +\max _{k \in J} \sum_{0<k_{\tau} \leq N-1} F\left(k, k_{\tau}\right)\left(\left|L-M_{\tau}\right|\left|q\left(k_{\tau}, y\left(k_{\tau}\right)\right)\right|+\left|\gamma_{\tau}\right|\right) \\
\leq & \frac{(l+H h)(N-m)}{1-(1-L)^{N}}+\frac{m}{1-(1-L)^{N}}\left(\left(L+\left|M_{\tau}\right|\right) h+\left|\gamma_{\tau}\right|\right)=\bar{\tau} .
\end{aligned}
$$

Based on the fact $0<L<1$, one has $1-(1-L)^{N}>0$. From Schaefer's fixed point theorem, we find that $\phi$ has at least one fixed point. So problem (3.7) has at least one solution.

Now, let $y$ be any solution of (3.7), we shall show that $y \in[v, w]$. First, we claim that $v(k) \leq y(k)$ for all $k \in J$. Taking $u(k)=v(k)-y(k), k \in J$, one has

$$
v(\theta(k))-q(\theta(k), y(\theta(k))) \geq \min _{k \neq k_{\tau}}\{u(\theta(k)), 0\}
$$

and

$$
v\left(k_{\tau}\right)-q\left(k_{\tau}, u\left(k_{\tau}\right)\right) \geq \min _{\tau=1}^{\tau}\left\{u\left(k_{\tau}\right), 0\right\} .
$$

Since $y(0)=y(N)$, we have $u(0)-u(N)=v(0)-v(N)$. From the definition of lower solution, we get

$$
\left\{\begin{array}{l}
\Delta u(k)+L u(k)+H \min \{u(\theta(k)), 0\} \leq-r_{u}, \quad k \neq k_{\tau}, k \in J^{\prime} \\
\Delta u\left(k_{\tau}\right) \leq-M_{\tau} \min \left\{u\left(k_{\tau}\right), 0\right\}-d_{u \tau}, \quad \tau=1,2, \ldots, m,
\end{array}\right.
$$

where $r_{v}, r_{u}, d_{v \tau}, d_{u \tau}$ are given by

$$
\begin{aligned}
& r_{v}=r_{u}= \begin{cases}\frac{L k+H \theta(k)+1}{N}[u(0)-u(N)], & \text { if } u(0)>u(N), \\
0, & \text { if } u(0) \leq u(N),\end{cases} \\
& d_{v \tau}=d_{u \tau}= \begin{cases}\frac{M_{\tau} k_{\tau}+1}{N}[u(0)-u(N)], & \text { if } u(0)>u(N), \\
0, & \text { if } u(0) \leq u(N) .\end{cases}
\end{aligned}
$$

From Lemma 2.5, we get $u(k) \leq 0$ on $J$, i.e., $v(k) \leq y(k), k \in J$. Analogously, one proves that $y(k) \leq w(k)$ on $J$ and $y \in[v, w]$.

Thus every solution $y$ lying between $v$ and $w$ of (3.7) is a solution of (3.1). Next, we shall show that the solution of (3.1) is unique. Assume that $y_{1}(k), y_{2}(k)$ are two solutions of (3.1). Putting $v_{1}(k)=u_{1}(k)-u_{2}(k), v_{2}(k)=u_{2}(k)-u_{1}(k)$, we get

$$
\begin{aligned}
& v_{1}(0)=v_{1}(N), \quad \Delta v_{1}(k)+L v_{1}(k)+H v_{1}(\theta(k))=0, \quad k \neq k_{\tau}, \\
& \Delta v_{1}\left(k_{\tau}\right)=-M_{\tau} v_{1}\left(k_{\tau}\right), \quad \tau=1,2, \ldots, m,
\end{aligned}
$$


and

$$
\begin{aligned}
& v_{2}(0)=v_{2}(N), \quad \Delta v_{2}(k)+L v_{2}(k)+H v_{2}(\theta(k))=0, \quad k \neq k_{\tau}, \\
& \Delta v_{2}\left(k_{\tau}\right)=-M_{\tau} v_{2}\left(k_{\tau}\right), \quad \tau=1,2, \ldots, m .
\end{aligned}
$$

From Lemma 2.5, we obtain $v_{1}(k) \leq 0, v_{2}(k) \leq 0$, and so $y_{1}(k)=y_{2}(k), k \in J$. This completes the proof.

Theorem 3.5 Assume that (2.1) holds, and

$\left(A_{0}\right)$ the functions $v, w \in \Omega$ are lower and upper solutions for (1.1), respectively, with $v \leq w$;

$\left(A_{1}\right)$ there exist $H \geq 0,0<L \leq M_{\tau}<1$ for $\tau=1,2, \ldots, m$, and a function $g \in C\left(J \times \mathbb{R}^{2}, \mathbb{R}\right)$ satisfying

$$
k(k, x, z)-g(k, u, s) \geq-L(x-u)-H(z-s)
$$

where $v(k) \leq y(k) \leq x(k) \leq w(k), v(\theta(k)) \leq s(\theta(k)) \leq z(\theta(k)) \leq w(\theta(k)), k \in J ;$

$\left(A_{2}\right) I_{\tau} \in C(\mathbb{R}, \mathbb{R})$ are functions satisfying

$$
I_{\tau}(u)-I_{\tau}(s) \geq-M_{\tau}(u-s)
$$

where $v\left(k_{\tau}\right) \leq s\left(k_{\tau}\right) \leq u\left(k_{\tau}\right) \leq w\left(k_{\tau}\right), \tau=1,2, \ldots, m$.

Then problem (1.1) has extremal solutions in the sector $[v, w]$.

Proof For any $\eta \in[v, w]$, we consider equations

$$
\left\{\begin{array}{l}
\Delta u(k)+L u(k)+H(\theta(k))=g(k, \eta(k), \eta(\theta(k)))+L(\eta(k))+H(\eta(\theta(k))) \\
\Delta u\left(k_{\tau}\right)+M_{\tau} u\left(k_{\tau}\right)=I_{\tau}\left(\eta\left(k_{\tau}\right)\right)+M_{\tau} \eta\left(k_{\tau}\right) \\
u(0)=u(N) .
\end{array}\right.
$$

Employing $\left(A_{1}\right),\left(A_{2}\right)$, Definition 3.1, and Definition 3.2, we have

$$
\begin{aligned}
& \Delta v(k)+L v(k)+H v(\theta(k)) \leq g(k, v(k), v(\theta(k)))-r_{v}+L v(k)+H v(\theta(k)) \\
& \leq g(k, \eta(k), \eta(\theta(k)))+L \eta(k)+H \eta(\theta(k))-r_{v}, \\
& \Delta v\left(k_{\tau}\right)+M_{\tau} v\left(k_{\tau}\right) \leq I_{\tau}\left(v\left(k_{\tau}\right)\right)-d_{\nu \tau}+M_{\tau} v\left(k_{\tau}\right) \\
& \leq I_{\tau}\left(\eta\left(k_{\tau}\right)\right)+M_{\tau} \eta\left(k_{\tau}\right)-d_{\nu \tau},
\end{aligned}
$$

where $r_{v}, d_{v \tau}$ are given by

$$
\begin{aligned}
& r_{v}= \begin{cases}\frac{L k+H \theta(k)+1}{N}[v(0)-v(N)], & \text { if } v(0)>v(N), \\
0, & \text { if } v(0) \leq v(N),\end{cases} \\
& d_{v \tau}= \begin{cases}\frac{M_{\tau} k_{\tau}+1}{T}[v(0)-v(N)], & \text { if } v(0)>v(N), \\
0, & \text { if } v(0) \leq v(N),\end{cases}
\end{aligned}
$$


and

$$
\begin{aligned}
& \Delta w(k)+L w(k)+H w(\theta(k)) \geq g(k, w(k), w(\theta(k)))+r_{w}+L w(k)+H w(\theta(k)) \\
& \geq g(k, \eta(k), \eta(\theta(k)))+L \eta(k)+H \eta(\theta(k))+r_{w}, \\
& \Delta w\left(k_{\tau}\right)+M_{\tau} w\left(k_{\tau}\right) \geq I_{\tau}\left(w\left(k_{\tau}\right)\right)+d_{w \tau}+M_{\tau} w\left(k_{\tau}\right) \\
& \geq I_{\tau}\left(\eta\left(k_{\tau}\right)\right)+M_{\tau} \eta\left(k_{\tau}\right)+d_{w \tau},
\end{aligned}
$$

where $r_{w}, d_{w \tau}$ are given by

$$
\begin{aligned}
& r_{w}= \begin{cases}\frac{L k+H \theta(k)+1}{N}[w(N)-w(0)], & \text { if } w(0)<w(N), \\
0, & \text { if } w(0) \geq w(N),\end{cases} \\
& d_{w \tau}= \begin{cases}\frac{M_{\tau} k_{\tau}+1}{N}[w(N)-w(0)], & \text { if } w(0)<w(N), \\
0, & \text { if } w(0) \geq w(N) .\end{cases}
\end{aligned}
$$

Observe that $v$ and $w$ are lower and upper solutions of (3.9), respectively. Noticing that (2.1) holds, by Theorem 3.4, one sees that (3.9) has exactly one solution $y \in \Omega$. Denote a map $A$ by $y(k)=A \eta(k)$. We shall prove that $A$ has the following properties:

(a) $v \leq A v, w \geq A w$;

(b) $A$ is a monotonically nondecreasing operator in $[v, w]$, i.e., for any $\eta_{1}, \eta_{2} \in[v, w]$, $\eta_{1} \leq \eta_{2}$ implies $A \eta_{1} \leq A \eta_{2}$.

For (a), we take $p(k)=v(k)-v_{1}(k)$, where $v_{1}=A v$. We distinguish two cases as follows.

Case 1: $v(0) \leq v(N)$. Following $\left(A_{0}\right)$ and $v_{1}(0)=v_{1}(N)$, we have

$$
\begin{aligned}
p(0)=v(0) & -v_{1}(0) \leq v(N)-v_{1}(N)=p(N), \\
\Delta p(k) \leq & g(k, v(k), v(\theta(k)))-\left[-L v_{1}(k)-H v_{1}(\theta(k))\right. \\
& +g(k, v(k), v(\theta(k)))+L v(k)+H v(\theta(k))] \\
\leq & -L p(k)-H p(\theta(k)), \quad k \neq k_{\tau}, k \in J^{\prime},
\end{aligned}
$$

and

$$
\Delta p\left(k_{\tau}\right) \leq-L_{k} p\left(k_{\tau}\right), \quad \tau=1,2, \ldots, m
$$

From Lemma 2.5, we have $p(k) \leq 0$ on $J$, i.e., $v \leq v_{1}$.

Case 2: $v(0)>v(N)$. By way of $\left(A_{0}\right)$, we derive

$$
\begin{aligned}
\Delta p(k) \leq & g(k, v(k), v(\theta(k)))-\frac{L k+H \theta(k)+1}{N}[v(0)-v(N)] \\
& -\left[-L v_{1}(k)-H v_{1}(\theta(k))+g(k, v(k), v(\theta(k)))+L v(k)+H v(\theta(k))\right] \\
\leq & -L p(k)-H p(\theta(k))-\frac{L k+H \theta(k)+1}{N}[p(0)-p(N)], \quad k \neq k_{\tau}, k \in J^{\prime},
\end{aligned}
$$


and

$$
\begin{aligned}
\Delta p\left(k_{\tau}\right)= & \Delta v\left(k_{\tau}\right)-\Delta v_{1}\left(k_{\tau}\right) \\
\leq & I_{\tau}\left(v\left(k_{\tau}\right)\right)-\frac{M_{\tau} k_{\tau}+1}{N}[v(0)-v(N)] \\
& -I_{\tau}\left(v\left(k_{\tau}\right)\right)-M_{\tau} v\left(k_{\tau}\right)+M_{\tau} v_{1}\left(k_{\tau}\right) \\
= & -M_{\tau} p\left(k_{\tau}\right)-\frac{M_{\tau} k_{\tau}+1}{N}[p(0)-p(N)], \quad \tau=1,2, \ldots, m .
\end{aligned}
$$

From Lemma 2.5, one gets $p(k) \leq 0$ for $k \in J$ and $v \leq v_{1}$.

On the basis of Case 1 and Case 2, we obtain $p(k) \leq 0$ for every $k \in J$. Hence $v \leq A v$.

Analogously, we have $w \geq A w$.

To prove (b), take $v_{1}=A \eta_{1}, v_{2}=A \eta_{2}$, where $\eta_{1} \leq \eta_{2}$ on $J$. Using $\left(A_{1}\right),\left(A_{2}\right)$, and (3.9), we get

$$
\begin{aligned}
\Delta p(k)= & \Delta v_{1}(k)-\Delta v_{2}(k) \\
= & {\left[-L v_{1}(k)-H v_{1}(\theta(k))+g\left(k, \eta_{1}(k), \eta_{1}(\theta(k))\right)+L \eta_{1}(k)+H \eta_{1}(\theta(k))\right] } \\
& -\left[-L v_{2}(k)-H v_{2}(\theta(k))+g\left(k, \eta_{2}(k), \eta_{2}(\theta(k))\right)+L \eta_{2}(k)+H \eta_{2}(\theta(k))\right] \\
\leq & -L\left(v_{1}(k)-v_{2}(k)\right)-H\left(v_{1}(\theta(k))-v_{2}(\theta(k))\right) \\
= & -L p(k)-H p(\theta(k)), \quad k \neq k_{\tau}, k \in J^{\prime}, \\
\Delta p\left(k_{\tau}\right)= & \Delta v_{1}\left(k_{\tau}\right)-\Delta v_{2}\left(k_{\tau}\right) \\
= & {\left[-M_{\tau} v_{1}(k)+I_{\tau}\left(\eta_{1}\left(k_{\tau}\right)\right)+M_{\tau} \eta_{1}\left(k_{\tau}\right)\right] } \\
& -\left[-M_{\tau} v_{2}\left(k_{\tau}\right)+I_{\tau}\left(\eta_{2}\left(k_{\tau}\right)\right)+M_{\tau} \eta_{2}\left(k_{\tau}\right)\right] \\
\leq & -M_{\tau}\left(v_{1}\left(k_{\tau}\right)-v_{2}\left(k_{\tau}\right)\right) \\
= & -M_{\tau} p\left(k_{\tau}\right), \quad \tau=1,2, \ldots, m,
\end{aligned}
$$

and $p(0)=p(N)$. In view of Lemma 2.5, we get $p(k) \leq 0$ on $J$, then $A \eta_{1} \leq A \eta_{2}$.

Now we define the sequences $\left\{v_{j}(k)\right\},\left\{w_{j}(k)\right\}$ with $v_{0}=v, w_{0}=w$ such that $v_{j}=A v_{j-1}$, $w_{j}=A w_{j-1}$. Due to (a) and (b), one reaches

$$
v_{0} \leq v_{1} \leq v_{2} \leq \cdots \leq v_{j} \leq \cdots \leq w_{j} \leq \cdots \leq w_{2} \leq w_{1} \leq w_{0} \quad \text { on } J .
$$

Hence, there exist $\rho(k)$ and $r(k)$ such that $\lim _{j \rightarrow \infty} v_{j}(k)=\rho(k)$ and $\lim _{j \rightarrow \infty} w_{j}(k)=r(k)$ uniformly on $J$.

Observe that $v_{j}(k), w_{j}(k)(j=1,2, \ldots)$ satisfy

$$
\left\{\begin{array}{l}
\Delta v_{j}(k)+L v_{j}(k)+H v_{j}(\theta(k)) \\
\quad=g\left(k, v_{j-1}(k), v_{j-1}(\theta(k))\right)+L\left(v_{j-1}(k)\right)+H\left(v_{j-1}(\theta(k))\right), \quad k \neq k_{\tau}, k \in J^{\prime}, \\
\Delta v_{j}\left(k_{\tau}\right)+M_{\tau} v_{j}\left(k_{\tau}\right)=I_{\tau}\left(v_{j-1}\left(k_{\tau}\right)\right)+M_{\tau} v_{j-1}\left(k_{\tau}\right), \quad \tau=1,2, \ldots, m, \\
v_{j}(0)=v_{j}(N),
\end{array}\right.
$$


and

$$
\left\{\begin{array}{l}
\Delta w_{j}(k)+L w_{j}(k)+H w_{j}(\theta(k)) \\
\quad=g\left(k, w_{j-1}(k), w_{j-1}(\theta(k))\right)+L\left(w_{j-1}(k)\right)+H\left(w_{j-1}(\theta(k))\right), \quad k \neq k_{\tau}, k \in J^{\prime}, \\
\Delta w_{j}\left(k_{\tau}\right)+M_{\tau} w_{j}\left(k_{\tau}\right)=I_{\tau}\left(w_{j-1}\left(k_{\tau}\right)\right)+M_{\tau} w_{j-1}\left(k_{\tau}\right), \quad \tau=1,2, \ldots, m, \\
w_{j}(0)=w_{j}(N) .
\end{array}\right.
$$

Obviously, $\rho(k)$ and $r(k)$ are solutions of (1.1).

We shall show that $\rho(k), r(k)$ are minimal and maximal solutions, respectively, of problem (1.1). Let $y(k)$ be any solution of problem (1.1) such that $v(k) \leq y(k) \leq w(k)$. Assume that there is a positive integer $j$ such that $v_{j}(k) \leq y(k) \leq w_{j}(k)$ on $J$. Based on the monotonically nondecreasing property of $A$, we easily get $v_{j+1}=A v_{j} \leq A y=y, v_{j+1}(k) \leq y(k), k \in J$. Analogously, one derives $y(k) \leq w_{j+1}(k)$ on $J$. For $v_{0}(k) \leq y(k) \leq w_{0}(k)$, by induction we see that $v_{j}(k) \leq y(k) \leq w_{j}(k), k \in J$. Let $j \rightarrow \infty$, we have $\rho(k) \leq y(k) \leq r(k)$ on $J$. This completes the proof.

Example 3.6 Consider the following equations:

$$
\left\{\begin{array}{l}
\Delta u(k)=-u^{2}(k)-\frac{1}{20} u\left(\frac{1}{2} k\right)+\frac{2^{-k}}{16}, \quad k \in Z[0,3], k \neq k_{1} \\
\Delta u\left(k_{\tau}\right)=-\frac{1}{2} u\left(k_{1}\right), \quad k_{1}=2 \\
u(0)=u(3)
\end{array}\right.
$$

Let $g(k, u(k), u(\theta(k)))=-u^{2}(k)-\frac{1}{20} u\left(\frac{1}{2} k\right)+\frac{2^{-k}}{16}$. It is easy to verify that $v=-\frac{1}{21}$ is a lower solution, and $w_{1}(k)=\frac{1}{24}\left(6-2^{-k}\right)$ is an upper solution for (3.10). Indeed,

$$
\begin{aligned}
w_{1}(0)= & \frac{1}{24}(6-1)<\frac{1}{24}\left(6-2^{-3}\right)=w_{1}(3), \\
\Delta w_{1}(2) & =\frac{1}{24}\left(2^{-2}-2^{-3}\right)>-\frac{1}{2} \cdot \frac{1}{24}\left(6-2^{-2}\right)+\frac{2}{3} \cdot \frac{1}{24} \cdot\left(1-2^{-3}\right), \\
\Delta w_{1}(k) & =\frac{1}{24}\left(2^{-k}-2^{-k-1}\right) \\
\geq & -\left[\frac{1}{24}\left(6-2^{-k}\right)\right]^{2}-\frac{1}{20} \cdot \frac{1}{24}\left(6-2^{-k}\right)+\frac{1}{16} \cdot 2^{-k}+\frac{19 k+40}{120} \cdot \frac{1}{24} \cdot \frac{7}{8},
\end{aligned}
$$

and

$$
g(k, x, y)-g(k, u, v)=-\left(x^{2}-u^{2}\right)-\frac{1}{20}(y-v) \geq-\frac{1}{2}(x-u)-\frac{1}{20}(y-v)
$$

for $v \leq u \leq x \leq w$. Putting $L=\frac{49}{100}, H=\frac{1}{20}, M_{\tau}=\frac{1}{2}$, we obtain

$$
\begin{aligned}
& \frac{1}{20} \sum_{i=0, i \neq k_{\tau}}^{3} \prod_{i<k_{\tau}<3}\left(1-M_{\tau}\right)(1-L)^{-\frac{1}{2} i-1}-\prod_{\tau=1}^{m}\left(1-M_{\tau}\right) \\
& \quad=\frac{1}{20} \sum_{i=0, i \neq k_{\tau}}^{3} \prod_{i<k_{\tau}<3} \frac{1}{2} \cdot\left(\frac{51}{100}\right)^{-\frac{1}{2} i-1}-\left(1-\frac{1}{2}\right) \\
& \quad=\frac{1}{20} \sum_{i=0}^{1}\left(\frac{51}{100}\right)^{-\frac{1}{2} i-1}+\frac{1}{20} \sum_{i=2}^{3} \frac{1}{2} \cdot\left(\frac{51}{100}\right)^{-\frac{1}{2} i-1}-\frac{1}{2}<\frac{1}{2}-\frac{1}{2}=0,
\end{aligned}
$$


which shows that all of the conditions of (2.1) are satisfied. Therefore, from Theorem 3.5, we arrive at the existence of extremal solutions of $(3.10)$ in $[v, w]$.

\section{Conclusion}

The theme of the paper is a study on periodic boundary value problems for first-order impulsive delay difference equations. It is well recognized that the theory of impulsive equations offers a general framework for the mathematical modeling of many real-world phenomena where the states undergo abrupt changes. Such equations have extensive applications in economics, dynamic systems, optimal control, medicine, population dynamics, and many other fields. In particular, in recent years, there has been an increasing interest in extending impulsive differential equations to time-delay systems and boundary value problems. On the other hand, difference equations play an important role in many fields such as numerous settings and forms, computing, electrical circuit analysis, biology, etc. However, there are not many related results for impulsive difference equations and impulsive delay difference equations. These motivated us to work on the present topic.

In this paper, we studied impulsive delay difference equations with periodic boundary conditions. Based on the new concepts of lower and upper solutions, we established two new comparison principles. With these, we constructed monotone sequences from a corresponding linear equation and established the existence of extremal solutions by utilizing the monotone iterative technique. An example was given to illustrate the results obtained. It is reckoned that these results may play an important role in the theory of difference equations, and are useful in many practical problems in the aforesaid fields.

\section{Acknowledgements}

The authors would like to thank the reviewers and the editors for their valuable suggestions and comments.

\section{Funding}

This work was supported by the NNSF of China (No. 11271106), the Fundamental Research Funds for the Central Universities (No. 2015ZD29, 13ZD19), the Higher School Science Research Funds of Hebei Province, China (No. Z2015137), and an Outstanding Teaching Award of the University of Hong Kong.

\section{List of abbreviations}

$\mathbb{R}:=$ the set of all real numbers; $\mathbb{N}:=$ the set of all natural numbers; $J:=Z[0, T]=\{0,1, \ldots, T\}$, where $T$ is a positive integer; $J^{\prime}:=Z[0, T-1]=\{0,1, \ldots, T-1\} ; \Omega:=$ the set of real-valued functions defined on $J ;\|y\|:=\max _{n \in J}|y(n)|$ for any $y \in \Omega$; $[v, w]:=\{y \in \Omega: v(n) \leq y(n) \leq w(n), n \in J\}$.

Availability of data and materials

Not applicable.

\section{Competing interests}

The authors declare that they have no competing interests.

Authors' contributions

The three authors investigated the problem separately at the beginning and got together to discuss, cross-fertilize, and came up with the final findings. They have equal share of contribution to the results. All three authors read and approved the final manuscript.

\section{Author details}

${ }^{1}$ College of Science and Technology, North China Electric Power University, Baoding, People's Republic of China. ${ }^{2}$ Department of Information Engineering, China University of Geosciences Great Wall College, Baoding, People's Republic of China. ${ }^{3}$ Department of Mathematics, University of Hong Kong, Pokfulam, Hong Kong.

\section{Publisher's Note}

Springer Nature remains neutral with regard to jurisdictional claims in published maps and institutional affiliations. 


\section{References}

1. He, Z., Ge, W.: Monotone iterative technique and periodic boundary value problem for first order impulsive functional differential equations. Acta Math. Sin. 18(2), 253-262 (2002)

2. Ladde, G.S., Lakshmikantham, V., Vatsala, A.S.: Monotone Iterative Techniques for Nonlinear Differential Equations. Pitman, Boston (1985)

3. Li, J., Shen, J.: Periodic boundary value problem for delay differential equations with impulses. Comput. Math. Appl. 193(2), 563-573 (2006)

4. Liang, R., Shen, J.: Periodic boundary value problem for the first order impulsive functional differential equations. J. Comput. Appl. Math. 202(2), 498-510 (2007)

5. Luo, Z., Jing, Z:: Periodic boundary value problem for first-order impulsive functional differential equations. Comput. Math. Appl. 55(9), 2094-2107 (2008)

6. Pang, P.Y.H., Agarwal, R.P.: Periodic boundary value problems for first and second order discrete systems. Math Comput. Model. 16(10), 101-112 (1992)

7. De la Sen, M.: Stability of impulsive time-varying systems and compactness of the operators mapping the input space into the state and output spaces. J. Math. Anal. Appl. 321(2), 621-650 (2006)

8. De la Sen, M., Luo, N.: A note on the stability of linear time-delay systems with impulsive inputs. IEEE Trans. Circuits Syst. 50(1), 149-152 (2003)

9. Wang, H., Ding, C.: Impulsive control for differential systems with delay. Math. Methods Appl. Sci. 36(8), 967-973 (2013)

10. Zhao, J., Zhao, J., Ge, W.: Upper and lower solutions for m-point impulsive BVP with one-dimensional p-Laplacian. Discrete Dyn. Nat. Soc. 2013, Article ID 783509 (2013)

11. Bainov, D.D., Lakshmikantham, V., Simeonov, P.: Theory of Impulsive Differential Equations. World Scientific, Singapore (1989)

12. Atici, F., Eloe, P.: Initial value problems in discrete fractional calculus. Proc. Am. Math. Soc. 137(3), 981-989 (2009)

13. Elaydi, S.N.: An Introduction to Difference Equations. Undergraduate Texts in Mathematics. Spinger, New York (2005)

14. Keney, W.G., Peterson, A.C.: Difference Equations: An Introduction with Applications. Academic Press, Tokyo (2001)

15. Ardjouni, A., Djoudi, A.: Existence of positive periodic solutions for a system of nonlinear neutral difference equations with variable delay. J. Nonlinear Funct. Anal. 2016, Article ID 20 (2016)

16. He, Z., Zhang, X.: Monotone iterative technique for first order impulsive difference equations with periodic boundary conditions. Appl. Math. Comput. 156(3), 605-620 (2004)

17. Li, B., Song, Q:: Asymptotic behaviors of non-autonomous impulsive difference equation with delays. Appl. Math. Model. 35(7), 3423-3433 (2011)

18. Thandapani, E., Balasubramanian, V.: Some new oscillation criteria for nonlinear neutral delay difference equations. J. Nonlinear Funct. Anal. 2014, Article ID 7 (2014)

19. Wang, P., Tian, S., Wu, Y.: Monotone iterative method for first-order functional difference equations with nonlinear boundary value conditions. Appl. Math. Comput. 203(1), 266-272 (2008)

20. Wang, P., Wang, W.: Anti-periodic boundary value problem for first order impulsive delay difference equations. Adv. Differ. Equ. 2015(1), Article ID 93 (2015)

21. Wang, P., Zhang, J.: Monotone iterative technique for initial-value problems of nonlinear singular discrete systems J. Comput. Appl. Math. 221(1), 158-164 (2008)

22. Zhu, W.: Invariant and attracting sets of impulsive delay difference equations with continuous variables. Comput. Math. Appl. 55(12), 2732-2739 (2008)

23. Zhu, W., Xu, D., Yang, Z:: Global exponential stability of impulsive delay difference equation. Appl. Math. Comput. 181(1), 65-72(2006)

24. Zhuang, W., Chen, Y., Cheng, S.: Monotone methods for a discrete boundary problem. Comput. Math. Appl. 32(12), $41-49(1996)$

\section{Submit your manuscript to a SpringerOpen ${ }^{\circ}$ journal and benefit from:}

- Convenient online submission

Rigorous peer review

- Open access: articles freely available online

- High visibility within the field

- Retaining the copyright to your article

Submit your next manuscript at $\gg$ springeropen.com 